Service social

\title{
La réaction du RUFUTS au rapport de la Commission Rochon
}

\section{Marcelle Laforest, Gisèle Legault, Réjean Mathieu et Martin Poulin}

Volume 38, numéro 2-3, 1989

Les politiques sociales

URI : https://id.erudit.org/iderudit/706443ar

DOI : https://doi.org/10.7202/706443ar

Aller au sommaire du numéro

Éditeur(s)

École de service social de l'Université Laval

ISSN

1708-1734 (numérique)

Découvrir la revue

Citer cet article

Laforest, M., Legault, G., Mathieu, R. \& Poulin, M. (1989). La réaction du RUFUTS au rapport de la Commission Rochon. Service social, 38(2-3), 282-296.

https://doi.org/10.7202/706443ar

\section{Résumé de l'article}

Cet article commente d'abord la place faite au social dans le rapport Rochon et dans le système de services, et s'interroge sur la logique qui a présidé à la détermination de services sociaux dits prioritaires. Suivent des commentaires et propositions sur la formation des intervenants sociaux, la participation professionnelle et la recherche sociale. Finalement, après avoir scruté la dimension communautaire contenue dans le rapport, les auteurs procèdent à une brève analyse des propositions quant aux réformes structurelles préconisées par la Commission. 
LAFOREST, Marcelle, professeure à

l'École de service social, Université Laval.

LEGAULT, Gisèle, professeure à l'École de service social, Université de Montréal.

MATHIEU, Réjean, professeur au Département de travail social, Université du Québec à Montréal. Poulin, Martin, professeur à l'École de service social, Université Laval.

\title{
La réaction du RUFUTS au rapport de la Commission Rochon
}

\author{
Marcelle Laforest \\ Gisèle Legault \\ Réjean Mathieu \\ Martin Poulin
}

Lors de leur congrès annuel du printemps 1988, les membres du Regroupement des unités de formation universitaire en travail social du Québec (RUFUTS) avaient procédé à l'étude du rapport de la Commission Rochon. Suite à cette analyse, les membres présents avaient exprimé le désir que leur organisme soit entendu dans le cadre de la tournée de consultation entreprise par la Ministre de la santé et des services sociaux après la parution dudit rapport. Ce souhait s'est réalisé et, le 28 juin 1988, un groupe porte-parole du RUFUTS dirigé par madame Christine Corbeil, coordonnatrice, rencontrait madame Lavoie-Roux et son groupe de travail pour livrer l'essentiel du message contenu dans un mémoire qui avait été préparé pour le RUFUTS par les membres de son Comité de la conjoncture.*

Reprenant l'essentiel de ce mémoire et des études du RUFUTS, le texte qui suit commente d'abord la place faite au social dans le rapport Rochon et dans le système de services et s'interroge sur la logique qui a présidé à la détermination de services sociaux dits 
prioritaires. Viennent ensuite des commentaires et propositions sur la formation des intervenants sociaux, la participation professionnelle et la recherche sociale. Finalement, après avoir scruté la dimension communautaire contenue dans le rapport, les auteurs procèdent à une brève analyse et font des propositions quant aux réformes structurelles préconisées par la Commission.

\section{Réaction globale et place du social dans le Rapport Rochon}

Notre position en est d'abord une d'appui aux travaux de la Commission qui jouit selon nous d'une grande crédibilité en raison du sérieux des études conduites et du nombre de personnes et d'organismes consultés. Nous sommes heureux que, dans I'ensemble, la Commission Rochon s'inscrive en continuité de la Commission Castonguay-Nepveu et qu'elle ne remette pas en question les grandes orientations de cette dernière, principalement la responsabilité de l'État en matière de services de santé et de services sociaux et l'accessibilité universelle et gratuite à ces services.

La Commission Rochon analyse l'état actuel des services de santé et des services sociaux en tenant compte de l'évolution des problèmes de santé et des problèmes sociaux. Nous sommes d'accord avec cette perspective qui a l'avantage de reconnaître une meilleure visibilité à la dimension sociale, tout en conférant à la dimension santé la place qui lui revient dans une conception de société moderne.

Cette position a surtout le mérite d'élargir le réservoir des paramètres qui devraient servir à la définition du concept de santé pour inclure l'influence des conditions sociales et psychosociales d'existence des populations. À cet égard, l'introduction de concepts comme ceux de satisfaction, de qualité de vie et de bien-être témoigne de l'envergure que pourrait prendre l'élaboration d'une politique globale de bien-être (ou de santé) fondée sur l'interaction individu-environnement.

Ce sont là des perspectives auxquelles nous souscrivons parce qu'elles sont porteuses de changement pour l'avenir. Car il faut bien noter qu'au-delà de l'état de question général portant sur les problèmes et les besoins, la Commission perpétue malgré tout la position historique d'infériorité relative du secteur social par rapport au secteur de la santé dans l'ensemble du système, comme en font foi des indicateurs tels que l'accessibilité et le financement. 


\section{Les problèmes sociaux prioritaires}

Dans l'état de question qu'elle dresse des problèmes et des besoins en matière de services sociaux, la Commission centre son analyse sur les notions de protection, de vulnérabilité et de difficultés de la vie quotidienne. La gradation des risques à laquelle cette analyse donne lieu, sert à déterminer trois niveaux de problèmes sociaux, à savoir les problèmes sociaux fondamentaux, les conditions de vulnérabilité de certaines populations particulières et les problèmes reliés aux difficultés courantes du fonctionnement en société (voir tableau 1).

La Commission retient comme prioritaires, et les qualifie de fondamentaux, les problèmes de premier niveau pour leurs caractères de gravité, de danger, d'effets néfastes et d'incapacité de la part des populations à y faire face. Cette catégorie de problèmes donne lieu à des services sociaux dits essentiels et qui sont axés sur la protection à accorder aux groupes à risques.

Le RUFUTS entretient de sérieuses réserves sur cette décision qu'a prise la Commission de définir l'essentiel des services sociaux par une problématique de protection. Comme le dit fort justement notre collègue Frédéric Lesemann, " ...la décision de définir le cœur des services sociaux par une problématique de la protection consiste à étendre la logique de la loi de la Protection de la jeunesse à l'ensemble des populations traditionnellement clientes des services sociaux, définies maintenant comme groupes à risques $»{ }^{1} \mathrm{Et}$, de poursuivre notre collègue, "à défaut de murs, il faut des lois de protection $",{ }^{2}$ anticipant ainsi l'un des effets concrets de la désinstitutionnalisation et de la non-institutionnalisation des personnes âgées, des malades psychiatriques et autres groupes dits " à risques".

La logique de la protection appelle celle de la judiciarisation pour assurer l'application des services sociaux essentiels reliés à cette catégorie de problèmes dits fondamentaux. En fait, la logique judiciaire liée à la logique de la protection aura pour effet d'assurer le droit à des services qui deviendront ainsi " socialement requis " et, de ce fait, obligatoirement dispensés. Ce qui aura l'avantage, comme le dit bien Lesemann, "de mettre fin à l'érosion constante des services sociaux essentiels $»^{3}$ en les libérant de la clause liée aux ressources disponibles qui a été, jusqu'à maintenant, l'un des principaux déterminants de la dispensation des services sociaux. Toutefois, nous voulons attirer l'attention sur le fait que cela aura aussi pour effet de voir mobilisées en bloc les ressources professionnelles par les problématiques de protection. Ce qui aura pour conséquence de confiner les professionnels du social à un rôle de socio-techniciens 
TABLEAU 1

Niveaux d'analyse des problèmes sociaux et des populations concernées

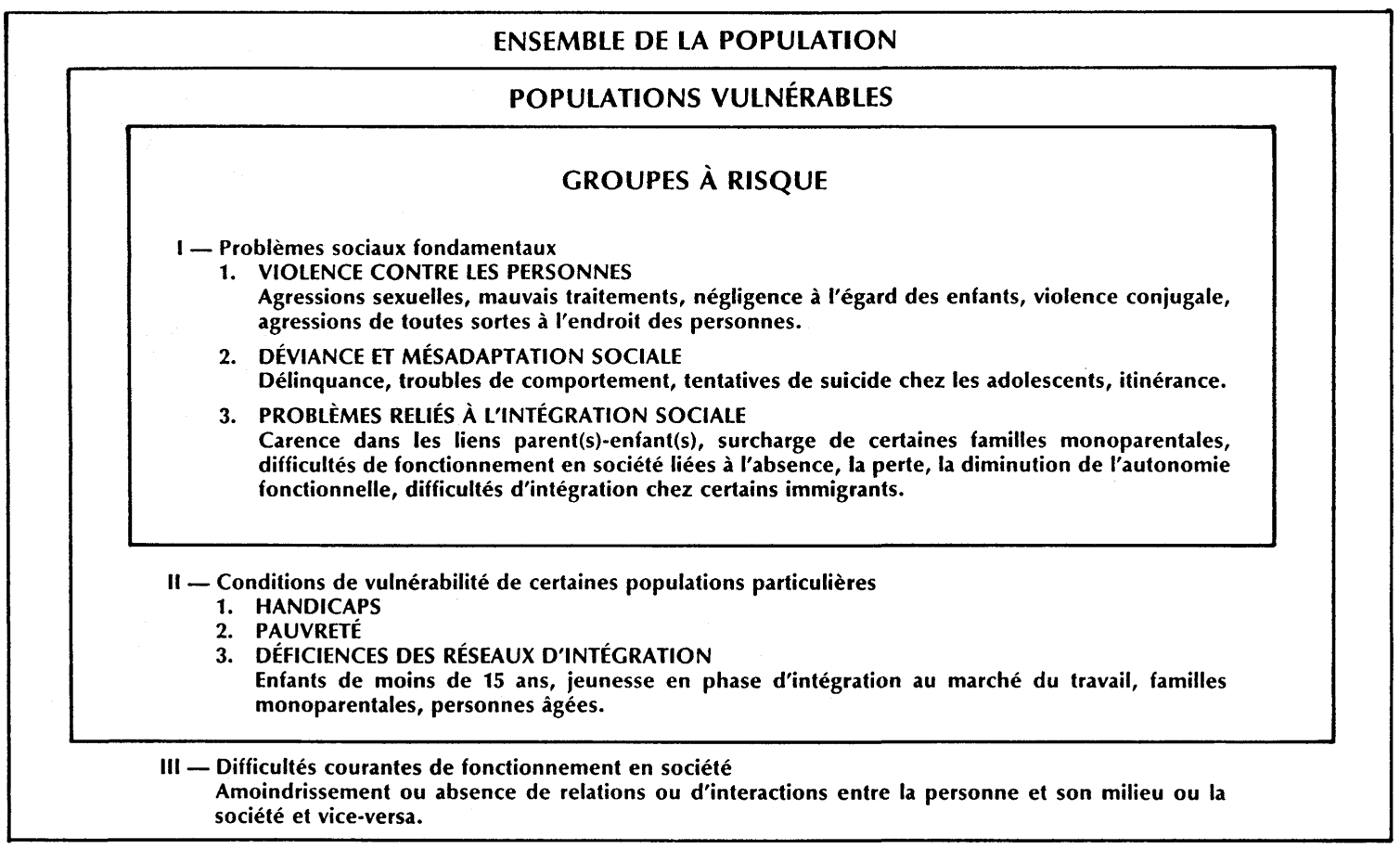


encadrés par des programmes prédéterminés, et donc privés d'une large part de leur autonomie d'intervenants.

D'autre part, les professionnels étant "réquisitionnés " par les programmes de protection, I'on confiera aux groupes communautaires les interventions "moins urgentes" et de type préventif requises par les deux autres niveaux de problèmes qui ne répondront sans doute pas aux critères du " socialement requis "; on peut penser que ce type d'intervention sera vraisemblablement soumis à « la règle des ressources disponibles". À noter que nous considérons très importante la présence des groupes communautaires dans la société comme nous jugeons indispensable leur action dans certaines problématiques sociales. Le danger que nous voyons, c'est que ces groupes œuvrant généralement sur une base bénévole et volontaire ne deviennent embrigadés dans la mission étatique et ne soient ainsi détournés de la fonction sociale qui leur est propre et combien nécessaire à la dynamique sociale.

Un autre effet limitatif que nous voyons au " socialement requis» tel que défini dans le Rapport, est relié celui-là à l'inaccessibilité structurelle qu'il semble receler. En ce sens que l'accessibilité aux services sociaux serait limitée et contrôlée par leur "caractère essentiel » de risque immédiat et apparent. Dans cette perspective, le caractère de vulnérabilité réservé aux deux autres catégories de problèmes, outre le fait qu'il est délicat d'en délimiter les contours, introduit dans les faits une forme de sélectivité qui aura probablement pour conséquence un transfert de clientèle du public au privé.

\section{Les ressources humaines}

\section{La formation}

En ce qui concerne la formation des ressources humaines, nous sommes d'avis qu'il doit certes y avoir adéquation entre les programmes de formation et les besoins du milieu, mais que la formation doit aussi fournir une analyse critique des pratiques et contribuer à les faire avancer. Les habiletés d'analyse critique acquises au cours de la formation lors des activités de recherche et d'intervention, doivent continuer de s'actualiser dans la pratique, ce qui nous apparait un gage de prévention de l'usure des professionnels. Cette caractéristique de la formation, conjuguée à une structure de gestion ouverte et à des opportunités de formation en cours d'emploi, dont nous traiterons plus loin, contribuerait à prévenir la fatigue et la démobilisation professionnelles dont il est fait mention dans le Rapport. 
Nous croyons aussi, comme nous le notions déjà à la Commission en $1986,{ }^{4}$ que la formation des intervenants sociaux doit être concertée à travers le Québec, entre autres à travers notre organisme; cette formation doit aussi être polyvalente en termes de problématiques, de clientèles et de méthodes d'intervention. Elle se doit d'être innovatrice, ne négligeant pas les approches préventives tout en demeurant en lien constant avec le milieu des services sociaux. Nous sommes aussi d'accord avec la Commission à l'effet qu'autant les professeurs que les établissements doivent se réapproprier leur responsabilité respective en ce qui a trait à la formation des intervenants sociaux.

Nous croyons que l'acte professionnel de l'intervenant social devrait être défini de façon plus rigoureuse; il ne doit pas l'être toutefois de façon trop restrictive et $n^{\prime}$ inclure que l'évaluation psychosociale. Il doit comprendre aussi, selon nous, les dimensions prévention et traitement des problèmes sociaux évalués, l'intervenant social étant, somme toute, l'un des spécialistes de la relation d'aide.

\section{La formation pratique}

Comme le suggère la Commission, universités et établissements doivent se réapproprier leur responsabilité respective en ce qui a trait à la formation pratique, trop souvent réduite aux seules activités de stages et dont la mise en œuvre est dévolue aux seuls responsables désignés pour s'occuper de ces activités. Il faudra aller beaucoup plus loin pour préciser comment cette formation pratique devra redevenir une responsabilité collective majeure. II faut en même temps éviter, dans le cas des universités, le piège de la formation à l'emploi et, dans le cas des établissements employeurs, celui de la « technicisation » de la fonction.

Nous endossons la proposition de la Commission à l'effet que "des mesures incitatives soient développées pour valoriser la participation des professeurs et des professionnels du réseau à la formation pratique des futurs professionnels et que les coûts liés aux stages de formation pratique soient intégrés aux budgets des établissements qui reçoivent les stagiaires $» .^{5}$ Mais certaines remarques s'imposent en regard du mode actuel de financement des stages.

La formule de facturation aux universités par les établissements selon le nombre de jours-stage effectués dans leur organisme, contribue largement à faire de la formation des étudiants un objet de marchandage de la part des organismes. D'une attitude antérieure de collaboration fondée sur l'échange de services entre milieux de pratique et universités, n'avons-nous pas adopté progressivement 
une attitude mercantile où l'étudiant est perçu comme un « mal nécessaire ", mais qui doit être rentable pour l'établissement?

L'attribution par le Ministère d'une responsabilité partagée universités-établissements concernant la formation pratique, assortie d'un financement adéquat des organismes, doit également s'accompagner de critères de qualité servant à sélectionner les lieux de formation accrédités par l'université. La formation pratique en service social ne peut s'inscrire à l'intérieur d'un programme universitaire sans cette garantie. Nous estimons aussi important de conserver la participation des groupes communautaires aux tâches de supervision; nous avons déjà une collaboration avec ces groupes et nous estimons précieux leur apport à la formation des étudiants.

Nous suggérerions dans un premier temps que le Ministère mette sur pied un comité d'étude sur la formation pratique en service social et son financement dans le but d'en arriver à une concertation universités-établissements-groupes communautaires.

\section{La formation en cours d'emploi}

"La formation en cours d'emploi et le perfectionnement dans le réseau des services de santé et des services sociaux sont des aspects négligés et sous-financés. " ${ }^{6} \|$ en est de même pour l'initiation à l'emploi. Ce constat de la Commission est tout à fait justifié. Comme institutions de formation, nous accueillons dans nos programmes des étudiants qui ne reçoivent aucun support de la part de leur organisme employeur. Ils assument eux-mêmes tout le fardeau de leur formation et de leur perfectionnement. Ils ne sont pas dégagés de leurs tâches. Ils doivent rembourser le temps qu'ils consacrent à leurs études et financer eux-mêmes tous les frais afférents à leur séjour à l'université (inscription, transport, frais de séjour, livres, services de secrétariat, etc.) Enfin, lorsque vient le temps de prévoir un stage de formation pratique, I'accueil est souvent tout aussi froid, si ce n'est pas un refus catégorique.

Ce que nous venons de décrire témoigne du désintéressement des établissements pour la formation et le perfectionnement de leur personnel professionnel, ce qui n'est pas étranger, selon nous, à la démotivation et à la démobilisation du personnel soulignées par la Commission. Cette dernière a dénoncé l'écart existant entre le secteur des services socio-sanitaires et les autres secteurs (particulièrement celui de l'éducation) concernant le pourcentage des dépenses affectées à la formation du personnel. La Commission ne s'est cependant pas rendue au bout de son analyse. Une recommandation spécifique concernant le pourcentage des dépenses (masses salariales) qui devrait être consacré à la formation aurait dû figurer 
dans le rapport. Le Ministère se doit de prendre position dans ce sens et proposer un accroissement progressif des budgets alloués à la formation du personnel. Nous suggérons un pourcentage variant progressivement entre $2 \%$ et $4 \%$.

\section{La participation professionnelle}

Les propositions de la Commission à ce chapitre sont intéressantes. Le type de gestion proposé et la philosophie qui le sous-tend méritent surtout d'être retenus. Comme modalité d'application, la Commission suggère la formation d'un comité consultatif à la direction. ${ }^{7}$ Cette nouvelle structure n'a que peu de chance de réussir si on tient compte du quasi-échec des comités consultatifs du personnel clinique dans bien des établissements.

Nous croyons qu'il serait préférable de privilégier la mise en place de groupes de qualité ou d'excellence, ou l'équivalent, dont la préoccupation principale serait I'amélioration des services et de la qualité des activités professionnelles. Ces groupes fonctionnent sur une base autonome et fondent leur action sur le renforcement de la responsabilité et de l'autonomie professionnelle. Des expériences de ce type sont en cours dans certains établissements et soulèvent l'intérêt et la participation d'un grand nombre de professionnels.

Ces groupes ne doivent cependant pas être une panacée pour résoudre les difficultés actuelles. Ils doivent plutôt s'intégrer dans une volonté réfléchie de l'établissement de donner à l'intervenant professionnel la place qui lui appartient dans l'organisation et de lui faire jouer un rôle correspondant à sa compétence et à son expertise. Ces groupes débouchent naturellement vers l'identification des besoins de formation et de perfectionnement professionnel auxquels l'établissement peut donner suite. On peut ainsi répondre collectivement et individuellement à ces besoins et les intégrer dans le développement du plan de carrière des personnes, tout en satisfaisant les besoins de l'établissement de rendre de meilleurs services, comme le souhaite la Commission. Il s'agit là, selon nous, d'une participation de concertation pour reprendre les termes de madame Lavoie-Roux dans sa lettre du 31 mai 1988 à notre organisme.

\section{La recherche sociale}

Nous sommes d'accord avec le diagnostic sévère de la Commission concernant ce sujet. ${ }^{8}$ Nous ne pouvons que souscrire à sa recommandation à l'effet que la recherche sociale puisse se développer. Nous avons déjà souligné, dans une prise de position antérieure 
auprès de la Commission, ${ }^{9}$ que nous trouvions très important : 1 . de développer des stratégies d'organisation de la recherche au niveau des unités de formation, en favorisant l'engagement et le regroupement des professeurs par problématiques spécifiques de recherche en lien avec les axes de formation et de pratiques sociales; 2. de développer des stratégies de financement de cette recherche par équipes de chercheurs-praticiens utilisant diverses approches en recherche tout en soutenant les projets d'évaluation des politiques et des pratiques sociales en cours.

Nous proposerions, pour démarrer les efforts du Ministère à ce chapitre, que soit institué un financement statutaire de la recherche sociale incluant une forme de discrimination positive pour un certain temps. Nous voulons aussi souligner l'importance d'une recherche sociale "libre» qui puisse coexister avec la recherche sociale orientée vers les priorités du réseau et du champ des affaires sociales. II nous apparaît particulièrement important de poursuivre entre autres la recherche sur le "socialement requis" et sur les " indicateurs de problèmes sociaux ».

\section{Le communautaire}

II nous semble important de souligner de prime abord que le Rapport Rochon constitue un progrès en ce qui a trait au communautaire, en particulier dans les C.L.S.C. Alors que le rapport Brunet le restreignait à l'intervention sur les conditions socio-sanitaires, la Commission Rochon ouvre la porte à la dimension socio-économique, ce qui nous semble hautement souhaitable dans la conjoncture actuelle surtout. Elle accorde également plus de place aux programmes locaux d'action communautaire, ce qui n'est pas moins souhaitable, en particulier dans une perspective de développement d'une plus grande prise en charge, localement, des besoins du milieu. ${ }^{10}$

En ce qui a trait aux organismes communautaires, il nous semble essentiel de reconnaître dans les faits "leur aptitude à mettre en lumière des problèmes mal connus ou ignorés " comme aussi " leur volonté (et nous ajouterions volontiers "leur capacité démontrée ») de participer activement à la définition des besoins et à l'élaboration des programmes, des politiques et des orientations gouvernementales ". ${ }^{11}$ Plus encore que les exemples cités par le rapport Rochon, qu'on se rappelle l'importance du rôle joué par les organismes communautaires dans les années 1960 pour renouveler les pratiques $\mathrm{d}^{\prime}$ intervention sociale, rôle confirmé amplement, ensuite, par la prise en chárge par l'État de nombreuses pistes d'intervention d'abord 
ouvertes par les organismes communautaires de l'époque... Qu'on pense aux cliniques populaires, juridiques ou de santé, à ces ancêtres des conseils régionaux qu'ont été les Conseils de développement social, et à un nombre important de regroupements divers de citoyens et citoyennes jusque-là sans voix dans le concert social, etc. $^{12}$

Or, les organismes communautaires qui sont de véritables expressions des besoins populaires et non des créations artificielles pour suppléer à des services publics, n'ont pas perdu, loin de là, cette qualité d'agir dans le sens d'un renouvellement des pratiques d'intervention sociale. Ils jouent un rôle prépondérant dans la création d'un nouveau tissu social, et ce, comme l'indique le rapport Rochon, «tant sur le plan thérapeutique que sur le plan de la participation à la vie démocratique ", en juxtaposant de "nouvelles solidarités communautaires aux réseaux traditionnels d'entraide $»{ }^{13}$ Nous faisons donc nôtres les préoccupations exprimées par la Commission autour de quatre grands enjeux dans ce domaine : " la reconnaissance du rôle et de la contribution des organismes communautaires, le respect de leur autonomie (garante de leur créativité spécifique, ajouterions-nous), un financement adéquat et la mise en œuvre d'une collaboration établie sur des bases équitables ${ }^{14}{ }^{14}$

Qu'il nous soit permis de souligner en particulier l'importance des organismes communautaires œuvrant auprès de populations comme les jeunes, les femmes, les personnes âgées, les personnes handicapées, ainsi que ceux qui favorisent le développement de rapports interculturels harmonieux, en raison de leur importance conjoncturelle particulière, auxquels nous ajoutons les organismes qui s'engagent dans le sens du développement économique communautaire, si important à la fois pour aller plus loin dans les possibilités de créativité populaire et pour lier l'économique au social et au culturel dans l'apprentissage collectif. Enfin, à ce niveau, il nous semble qu'une saine politique ne doit pas craindre d'encourager les organismes de pression, sources de prise de conscience de problèmes méconnus et d'éducation populaire importante.

\section{Financement des organismes communautaires}

En ce qui a trait au financement de ces organismes communautaires, qu'il nous soit permis de rappeler nos prises de position antérieures : une participation enrichissante à la dynamique sociale leur sera possible, d'une part, dans la mesure où leur financement de base ne grugera pas continuellement l'essentiel de leurs énergies et, d'autre part, dans la mesure où un financement supplémentaire accompagnerait la conception et la réalisation de projets novateurs. 
Nous pensons donc qu'il serait socialement utile d'inventorier davantage cette piste d'une subvention d'infrastructure et de fonctionnement de base, à laquelle pourrait être jointes des subventions non statutaires pour couvrir des services novateurs, voire dans certains cas, des expériences pilotes. Ajoutons enfin que dans le contexte actuel dominant, il nous semble que des projets axés sur la dimension "prévention » devraient tout particulièrement être encouragés.

\section{Rôle des organismes communautaires}

Si le Rapport Rochon accorde une place remarquable aux organismes communautaires, s'il insiste même, à certains moments, sur le fait que leur philosophie diffère de celle des établissements publics et qu'ils ne doivent donc pas être restreints à un rôle de suppléance, il nous semble pourtant les situer "ultimement dans le prolongement, le relais, la suppléance, la complémentarité des services sociaux existants. On est donc en présence d'une représentation hiérarchisée et complémentaire des services sociaux qui, partant des services de protection pour les groupes à risque, passe à des services sociaux généraux, accessibles en fonction de la précarité des ressources financières des bénéficiaires pour aboutir aux ressources communautaires vouées au développement social, à la prévention, à la promotion et à la défense des intérêts des personnes qu'ils représentent ou encore à la dispensation de services généraux à la communauté ${ }^{1}{ }^{15}$

$\mathrm{Qu}$ 'il nous soit permis, à ce sujet, de rappeler ce que nous disions déjà à l'item consacré aux services sociaux essentiels à l'effet que la perspective communautaire réelle qui en ressort risque de s'inscrire dans« un social en cascade ou, à l'inverse, en écluse, dans lequel retombe vers les mers communautaires tout ce que les canaux institutionnels $n^{\prime}$ ont pas été en mesure, ou n'ont pas jugé pertinent de retenir. " ${ }^{16}$

À notre avis, cette approche présente au moins deux dangers majeurs. D'une part, elle risque de cantonner le communautaire dans des services qui seront indirectement - et peut-être de plus en plus directement - déterminés par le réseau public, et d'autre part, celuici sera d'autant plus coupé des sources d'un véritable renouvellement permanent qu'il aura laissé au communautaire et au bénévolat "l'insaisissable, l'éphémère, l'imprévisible du social vivant ". ${ }^{17}$ Répétons-le, les groupes communautaires ne doivent pas être embrigadés dans la mission étatique : ils ont leur place spécifique irremplaçable dans la dynamique sociale, et l'État, pour sa part, ne doit surtout pas faire reposer sur eux des dimensions aussi importan- 
tes que la prévention et l'intervention au niveau de services même non-essentiels...

\section{Les structures et la participation des usagers}

II nous apparaît important d'indiquer notre adhésion générale à la nécessité de procéder à un nouveau partage des pouvoirs.

En effet, cette nécessité repose sur un diagnostic, à notre avis très juste, posé par la Commission Rochon, qui met en lumière la trop grande centralisation du processus décisionnel actuel.

II se pourrait bien, d'ailleurs, que ce diagnostic mérité explique en partie la résistance au Rapport qui a marqué la réaction de certains milieux. Pourtant, ce diagnostic nous interpelle fortement et il constitue vraisemblablement une condition sine qua non de toute réforme valable du système des services sociaux et de santé au Québec. Qu'on évacue cette partie du Rapport Rochon et on risque de reporter aux calendes grecques un renouvellement effectif des pratiques d'intervention sociale.

Nous avons déjà signalé, dans nos divers mémoires à la Commission Rochon, que l'actuelle centralisation ne nous semblait pas favoriser une réponse adéquate aux besoins locaux. Ainsi, on a pu voir à plusieurs reprises des C.L.S.C. proposer une programmation formulée dans le cadre de nombreuses consultations dans les milieux les plus représentatifs de leur clientèle et essuyer ensuite un refus généralement discutable au profit de politiques globales et uniformes. Ceci n'est qu'un exemple parmi d'autres, mais il nous semble probant, compte tenu du rôle spécifique des C.L.S.C. dans le système des services sociaux et de santé.

Bien sûr, le diagnostic n'est pas tout et la solution n'est pas aussi évidente. Nous pensons cependant que la régionalisation proposée par la Commission Rochon pourrait bien constituer un pas dans la bonne direction. Les régies régionales prévues, sans être des solutions miracles, auraient au moins l'avantage d'équilibrer davantage le balancier en faveur de débats ouverts sur les besoins spécifiques d'une région donnée, au lieu de laisser trop de décisions suivre la filière des groupes de pression les plus organisés et les plus proches du pouvoir quel qu'il soit. Les opposants à une telle décentralisation recourront probablement aux traditionnels arguments à l'effet que cette décentralisation risque de saper un nécessaire contrôle des fonds publics et que, de toutes façons, elle ne constituerait pas une véritable démocratisation. Outre le fait que le contrôle des fonds publics n'est pas nécessairement satisfaisant quelle que soit l'instance qui l'exerce, on peut au moins supposer que 
là où les décideurs sont plus proches des bénéficiaires des services, il existe au moins une plus grande possibilité d'expression efficace pour ceux-ci. Actuellement, la centralisation risque de permettre que les lobbyistes les plus efficaces, mais pas nécessairement les plus représentatifs ni les plus judicieux, l'emportent sur des organismes plus enracinés dans les besoins de la population mais moins habiles à faire valoir leurs points de vue. Enfin, comme la responsabilité financière doit normalement accompagner le pouvoir de décision, il nous apparaît souhaitable de pousser plus loin la réflexion sur l'autonomie de gestion des régies régionales avant d'en rejeter le principe.

En ce qui a trait au comité régional des priorités, il nous apparaît comme devant favoriser un nécessaire partage des points de vue en même temps que des expertises nécessaires à une prise de décision adéquate.

Dans l'ensemble donc, nous privilégions une décentralisation des pouvoirs parce qu'elle devrait faciliter une plus grande adéquation entre les besoins et les services en même temps qu'une meilleure intégration des services aux niveaux local et régional. Évidemment, cela suppose que l'instance régionale jouisse des pouvoirs inhérents à ses responsabilités, c'est-à-dire des fonctions reconnues de planification, de coordination, d'évaluation de programmes et d'allocation des ressources, quitte à revoir en conséquence les structures de négociation des conditions de travail, du moins sous certains aspects.

\section{La participation des usagers}

Nous sommes un peu insatisfaits des propositions de la Commission Rochon à cet égard, même si nous sommes d'accord avec une participation à la fois décisionnelle dans certains cas, consultative sur les orientations des services et de type "concertation"sur les besoins et préoccupations. Cela nous semble aller dans le sens d'une nécessaire restructuration de la participation démocratique. En effet, il faut éviter de privilégier des structures de participation factices, mais en même temps, il faut créer des mécanismes pouvant permettre une réelle participation. En ce sens, s'il nous semble toujours nécessaire d'assurer la présence de représentants d'usagers sur les instances de direction des établissements, même si ce n'était que pour "veiller au grain ", il nous apparaîtrait enrichissant pour les services et pour la démocratie de favoriser l'émergence et le fonctionnement systématique de regroupements d'usagers, évitant ainsi $d$ 'isoler des individus dans un cadre facilement obnubilant en accentuant au contraire l'échange et la prise de conscience collective. Une telle approche (non miraculeuse, nous le savons bien), serait 
également plus intéressante encore dans la mesure où il s'agirait de regroupements qui ne se cantonneraient pas à des tâches de critiques, mais ouverts également à des perspectives de création de services et de démarches collectives dépassant le cadre restreint de leur établissement. Enfin, là plus qu'ailleurs encore, la plus grande autonomie demeure nécessaire, permettant à ces regroupements de $s^{\prime}$ exprimer sans avoir peur de perdre leurs subventions.

\section{En guise de conclusion}

Enfin, nous voudrions souligner, comme la Commission, l'importance d'une approche sociale globale et préventive des problématiques fondée sur une constante complémentarité entre l'intervention individuelle et communautaire pour assurer un renouvellement continu des pratiques d'intervention sociale. Ceci suppose une communication étroite entre les professionnels et les groupes communautaires d'un milieu donné et un appui réel à ces groupes de la part des institutions, d'autant plus qu'ils ont souvent été le lieu de gestation de pratiques nouvelles, subséquemment endossées par les services publics. Une telle approche devrait être facilitée selon nous par une décentralisation administrative, et une gestion participative qui permette un efficace soutien aux employés, une possibilité réelle $\mathrm{d}^{\prime}$ encadrement professionnel, et enfin, une participation de ceux-ci aux délimitations de la charge de travail et à son évaluation; ceci serait de nature, croyons-nous, à contrer la démobilisation et la démotivation actuelles des ressources humaines. Nous soutenons également la position de la Commission en faveur de l'élaboration d'une politique de développement de ces ressources qui vienne contrer le sousdéveloppement actuel du secteur social.

Enfin, il est vrai que les propositions de la Commission concernant le financement des services nous laissent, comme tout le monde, sur notre faim. Cependant, il n'en est pas moins vrai qu'il est essentiel, dans la conjoncture actuelle, de réaffirmer la responsabilité de l'État, et donc son rôle dans le financement des services, alors que trop de gens se contentent actuellement de voir dans le financement privé la panacée à tous nos maux.

\section{Notes et références}

* Ce comité chargé d'étudier les principales questions conjoncturelles qui concernent la réalité de la formation universitaire en travail social au Québec se composait de Gisèle Legault, Marcelle Laforest, Réjean Mathieu, 
membres permanents du Comité et Martin Poulin, membre ad hoc. Coauteurs du présent texte, ils composaient le groupe porte-parole mentionné ci-avant.

${ }^{1}$ Frédéric Lesemann, "Le Rapport Rochon et le "social » : une interprétation ", Intervention, $\mathrm{n}^{\circ} 80$, juin 1988, p. 71.

2 Ibid.

${ }^{3}$ Ibid.

${ }^{4}$ RUFUTS, Annexe à "Une esquisse de notre problématique des services sociaux ", Synthèse et recommandations, juin 1986.

${ }^{5}$ Rapport de la Commission Rochon, p. 586.

${ }^{6} / d .$, p. 566.

7 Id., p. 569.

8 Id., p. 635.

${ }^{9}$ RUFUTS, op. cit.

${ }^{10}$ Rapport de la Commission Rochon, p. 522.

11 Id., p. 297.

12. L'histoire de la clinique des citoyens et citoyennes de Saint-Jacques est, à ce sujet, exemplaire : "Autrement dit, l'apparition de la Clinique s'inscrit dans I'histoire de la réforme moderne de la santé au Québec. Elle est liée au fait que des besoins exprimés dans la population en matière de santé n'étant pas encore satisfaits, un groupe de personnes s'est constitué en service public, s'est, en somme, substitué à l'Etat. Lorsque ces grandes réformes sociales dans la santé ont été pour l'essentiel complétées, la continuation de la Clinique est devenue pratiquement impossible " (Boivin, Robert, L'histoire de la clinique des citoyens de Saint-Jacques, 1968-1988, Montréal, VLB éditeur, 1988.

Pourtant, comme un employé maintenant rattaché au C.L.S.C. I'indiquait : "Ici, nous sommes 120, les bureaux sont cloisonnés, il y a des employés que je ne connais même pas. La différence avec la Clinique est propre à la structure, qui tue dans l'œuf le sentiment d'appartenance, l'initiative, la spontanéité, l'implication. On devient fonctionnaire insidieusement " (même source).

${ }^{13}$ Rapport de la Commission Rochon, p. 297.

14 Id., p. 298.

${ }^{15}$ Frédéric Lesemann, op. cit., p. 73.

16 Id., p. 74.

17 Ibid. 